\title{
An Improved 6S Code for Atmospheric Correction Based on Water Vapor Content ${ }^{*}$
}

\author{
Yin Zhang, Xiaoqin Wang, Yunzhi Chen \\ Key Laboratory of Data Mining \& Information Sharing of Ministry of Education, Fuzhou University, Fuzhou, China \\ Email: zhangy910@foxmail.com
}

Received April 18, 2012; revised May 29, 2012; accepted June 15, 2012

\begin{abstract}
Water vapor content in the atmosphere is very significant for atmospheric correction of optical remote sensing data. Nowadays, the common atmospheric correction models use a single value of the average water vapor content of the study area to perform atmospheric correction. As the distribution of water vapor content varies greatly with time and space, it is obviously inaccurate to represent the total water vapor conditions of the whole area by just reading the average water vapor content. In this study, we altered the $6 \mathrm{~S}$ sources so that it could read the water vapor content image which was retrieved from MODIS $1 \mathrm{~km}$ data. Atmospheric correction was implemented for the band 1 of MODIS $500 \mathrm{~m}$ data pixel-by-pixel using the improved $6 \mathrm{~S}$ model. In comparison with the traditional $6 \mathrm{~S}$ model, this improved $6 \mathrm{~S}$ model is more reasonable in atmospheric correction, for it considers the spatial distribution of the water vapor content retrieved from MODIS data in the near infrared to define the atmospheric conditions for simulating the atmospheric radiative transfer. The results corrected by the improved $6 \mathrm{~S}$ model showed more reasonable in pixel spatial distribution and closer histogram with the original image than those by traditional $6 \mathrm{~S}$ model.
\end{abstract}

Keywords: Atmospheric Correction; 6S Model; Water Vapor Content; MODIS

\section{Introduction}

Since the first Earth's digital image was acquired, scientists have shown great interest in atmospheric correction [1]. There appears many atmospheric correction methods during the 40 years' development, the kind of method, take 6S [2], MODTRAN [3] and LOWTRAN [3] for instance, which is based on radiative transfer (RT) code is of high accuracy among them. One should estimate the atmospheric parameters before using these methods. The temperature, the pressure, the water vapor and ozone content and the aerosol optical depth are the necessary parameters, because the program will perform atmospheric correction by using them to simulate the transmission conditions of the solar radiation in the atmosphere. In the actual application, however, it seems very hard to obtain the atmospheric parameters synchronously with the image, especially the retrieval of the water vapor content and the aerosol optical depth (AOD), because the distribution of water vapor and aerosol varies greatly with time and space [4]. Water vapor plays an important role in atmospheric processes, which has an impact on the measured radiance because of its absorption in the solar spectrum. It is necessary to choose a standard at-

\footnotetext{
*Natural Science Foundation for Distinguished Young Scholars of Fujian Province (Grant No. 2009J06024).
}

mospheric model or to enter the water vapor and ozone content to define the atmospheric conditions in the atmospheric correction models in common use. Thus, the remote sensing image covers vast with a large space span, especially the low resolution images, single value of the average water vapor content cannot satisfy with the accurate description of the atmospheric conditions of the whole area.

It will be possible to derive the water vapor content from Terra MODIS by using the "water vapor" channels in addition to the existing "window" channels. Terra was launched on Dec. 12, 1999 and flies northward pass the equator at about local time 10:30 AM, which can provide the synchronous or quasi-synchronous spatial distributions of the water vapor content for atmospheric correction. Kaufman and Gao (1992) [5], Sobrino and Kharraz (2003) [6] utilized the ratios of bands radiation to estimate water vapor content. Mao and $\mathrm{Li}$ (2010) [7] used the RT and neural network (NN) to estimate the water vapor content.

6S is widely used today, which is based on RT theories. Zhao and Tamura (2000) [8] chose a standard atmospheric model and a standard aerosol model for atmospheric correction based on $6 \mathrm{~S}$ code. In this study, $6 \mathrm{~S}$ code was improved to read the water vapor content image which was retrieved from the EOS/MODIS data for 
atmospheric correction of the optical remote sensing data.

\section{Methodology}

The flow chart of the atmospheric correction method presented in this paper is shown in Figure 1. The total water vapor content was retrieved from MODIS data with $1 \mathrm{~km}$ resolution at first. Then, $6 \mathrm{~S}$ code was improved so that it can read the water vapor content image. At last the atmospheric parameters were obtained and atmospheric correction was implemented. The keys are to improve the $6 \mathrm{~S}$ code and estimate the water vapor content.

\subsection{Atmospheric Radiative Transfer Theory and 6S Model}

In the solar spectrum, sensors on Earth remote sensing satellites measure the radiance reflected by the Atmosphere-Earth surface system. This signal mainly depends on the surface reflectance but is also perturbed by the gaseous absorption and the scattering by molecule and aerosols. The measured quantities can be expressed in terms of apparent reflectance $\rho^{*}$, defined as

$$
\rho^{*}=\pi L / \mu_{s} E_{s}
$$

where $L$ is the measured radiance, $E_{s}$ is the solar flux at the top of the atmosphere and $\mu_{s}=\cos \left(\theta_{s}\right)$ where $\theta_{s}$ is the sun zenith angle.

Think of the lambertian uniform target with $\rho_{s}$, the apparent reflectance can be written [9]:

$$
\rho^{*}=\rho_{a}+T\left(\theta_{s}\right) T\left(\theta_{v}\right) \frac{\rho_{s}}{1-S \rho_{s}}
$$

where $\rho_{a}$ refers to the intrinsic atmospheric reflectance, $T\left(\theta_{s}\right)$, [respectively $T\left(\theta_{v}\right)$ ] corresponds to the total transmission of the atmosphere on the path between the sun and the surface, (respectively between the surface and the sensor). $S$ is the spherical albedo of the atmosphere.

In actual fact, the surface is non-uniform, we should consider the environment effects on the radiative transmission, and the apparent reflectance can be written [9]:

$$
\begin{aligned}
\rho^{*}= & \rho_{a}+\frac{T\left(\theta_{s}\right)}{1-<\rho_{s}(M)>S} \\
& \cdot\left(\rho_{s}(M) e^{-\tau / \mu v}+\left\langle\rho_{s}(M)\right\rangle t_{d}\left(\theta_{v}\right)\right)
\end{aligned}
$$

where $\rho_{s}(M)$ corresponds to the reflectance of the target $M$ and $<\rho_{s}(M)$ corresponds to the reflectance of the uniform environment, $t_{d}\left(\theta_{v}\right)$ is the upward diffuse transmittance factor and $e^{-\tau / \mu v}$ is the upward direct transmittance factor.

The original $6 \mathrm{~S}$ is an improved version of $5 \mathrm{~S}$. It enables to simulate plane observation, to account for elevated targets, to take into account non lambertian surface

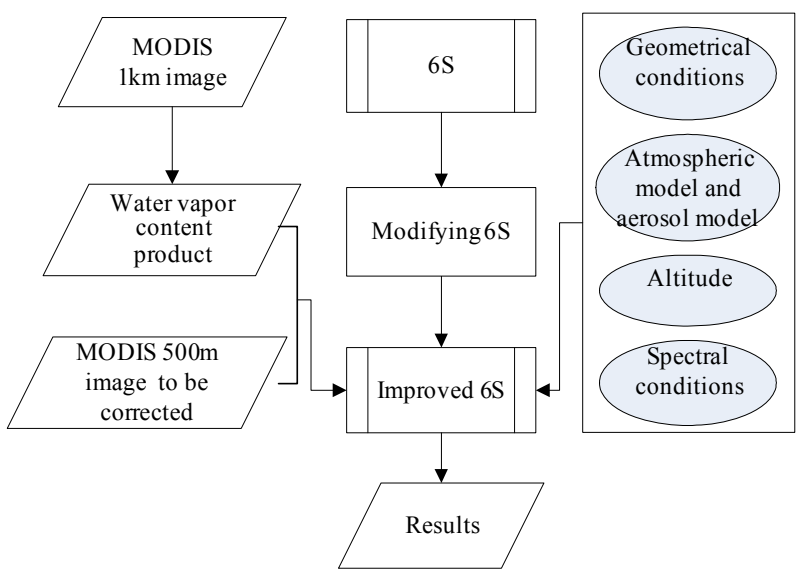

Figure 1. Flow chart of the atmospheric correction.

conditions, and new gases $\left(\mathrm{CH}_{4}, \mathrm{~N}_{2} \mathrm{O}, \mathrm{CO}\right)$ have been integrated in the computation of the gaseous transmission. The computational accuracy for Rayleigh and aerosol scattering effects has been improved by the use of state-of-the-art approximations and implementation of successive orders of scattering (SOS) algorithm. The step size used for spectral integration has been improved to $2.5 \mathrm{~nm}$. It provides some kinds of standard atmosphere models and aerosol models; in addition, people can customize a model as well.

This study utilized the $6 \mathrm{~S}$ code extended by Mauro Antunes who is a professor in Brazil, hereinafter to be referred as Antunes's 6S. Though Antunes's 6S model reads and writes only RAW images of 8 bits, the input mode of water vapor content is not altered. In view of this, Antunes's $6 \mathrm{~S}$ code was improved to read the spatial distribution of water vapor content and the input image synchronously in our study. The other atmospheric parameters need not to be changed. Meanwhile, the value of the samples and lines of the image should also be given as input parameter.

\subsection{Estimation of Water Vapor Content}

Water vapor content is indispensable for atmospheric correction. The near-infrared at around $1 \mu \mathrm{m}$ is sensitive to water vapor, so water vapor content can be retrieved from EOS/MODIS in the Near IR. In the paper, the algorithm developed by Sobrino (2003) [6] was chosen for water vapor content estimation. The implementation of the algorithm is very simple and includes three basic steps:

Firstly, computing the ratios between bands 17, 18, 19 and 2:

$$
G_{17}=L_{17} / L_{2}, G_{18}=L_{18} / L_{2}, G_{19}=L_{19} / L_{2}
$$

where $L_{i}$ is the measured radiance of the $i^{\text {th }}$ band.

Secondly, computing the water vapor content in each "water vapor" channel: 


$$
\begin{aligned}
& W_{17}=26.314-54.434 G_{17}+28.449 G_{17}^{2} \\
& W_{18}=5.012-23.017 G_{18}+27.884 G_{18}^{2} \\
& W_{19}=9.446-26.887 G_{19}+19.914 G_{19}^{2}
\end{aligned}
$$

where $W_{i}$ refers to the water vapor content in the $i^{\text {th }}$ band, $i=17,18$ or 19 .

Finally, calculating the total water vapor content by giving a weight to each channel:

$$
W=0.192 W_{17}+0.453 W_{18}+0.355 W_{19}
$$

where $W$ corresponds to the total water vapor content.

\section{Atmospheric Correction}

\subsection{Study Site}

The data we used is MODIS multi-spatial scale data with resolution $500 \mathrm{~m}$ and $1 \mathrm{~km}$. The original image size is $560 \times 480$ pixels with resolution $500 \mathrm{~m}$, covering the city of Fuzhou, in Fujian province, China. MODIS imaging date is 25 th, May, 2010. The gray image of MODIS band 1 with resolution $500 \mathrm{~m}$ was shown in the left of Figure 2.

Water vapor content retrieval experiments were carried out from MODIS with resolution $1 \mathrm{~km}$ using the method introduced above. The estimated water vapor content image was shown in the right of Figure 2.

\subsection{Model Implementation}

Before running the improved 6S model, we should obtain the atmospheric parameters first. The main parameters are as follows:

- Geometrical conditions. To define geometrical conditions when using MODIS data, input values for month, day, universal time, center latitude and longitude of the image were entered. The code would automatically estimate the solar zenith angle, view zenith angle and the solar and view azimuth angle.

- Atmospheric model. There are 7 standard atmospheric models and 2 user-defined models proposed by $6 \mathrm{~S}$. We used the latter with assuming that the ozone content was $0.3 \mathrm{~cm} / \mathrm{atm}$.

- Aerosol model (type and concentration). We chose the continental model to define aerosol type. To define
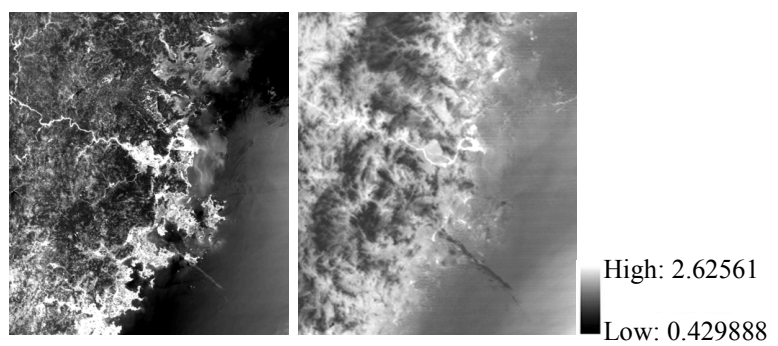

Figure 2. Image to be corrected (left) and water vapor content (right) $\left(\mathrm{g} / \mathrm{cm}^{2}\right)$. the concentration of aerosols, visibility $(\mathrm{km})$ should be entered directly into $6 \mathrm{~S}$. The aerosol optical thickness at $550 \mathrm{~nm}$ should then be computed from a standard aerosol profile by using subroutine ODA550 provided by $6 \mathrm{~S}$.

- The altitude of the target and the sensor. We assumed that the target is at the sea level and the sensor is out of the atmosphere.

- The spectral conditions. We chose to enter the wavelength range of MODIS band1.

Using the data and parameters above, the improved $6 \mathrm{~S}$ code was implemented for atmospheric correction of the MODIS band1 with resolution $500 \mathrm{~m}$. Meanwhile, the Antunes's 6S code was also performed in the same conditions with the average water vapor content of 0.9843 $\mathrm{g} / \mathrm{cm}^{2}$.

\section{Results and Discussion}

The two corrected images were shown in Figure 3. The remarkable differences can be seen between the two images. There are apparent stripes in the left image corrected by Antunes's 6S model. Therefore, we can infer that the right image which was corrected by the improved $6 \mathrm{~S}$ model is more reasonable in the spatial distribution than the left image, especially in the ocean area. Then histograms (Figure 4) and difference images (Figure 5)
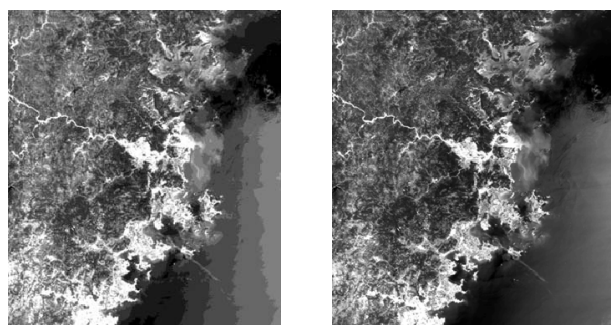

Figure 3. Image corrected by Antunes's 6S (left) and image corrected by improved 6S (right).

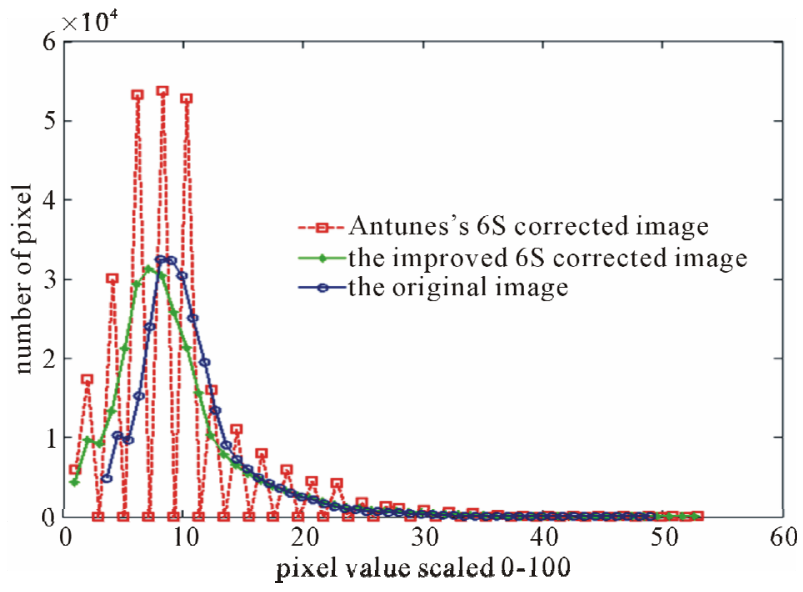

Figure 4. Histograms of the three images before and after atmospheric correction. 

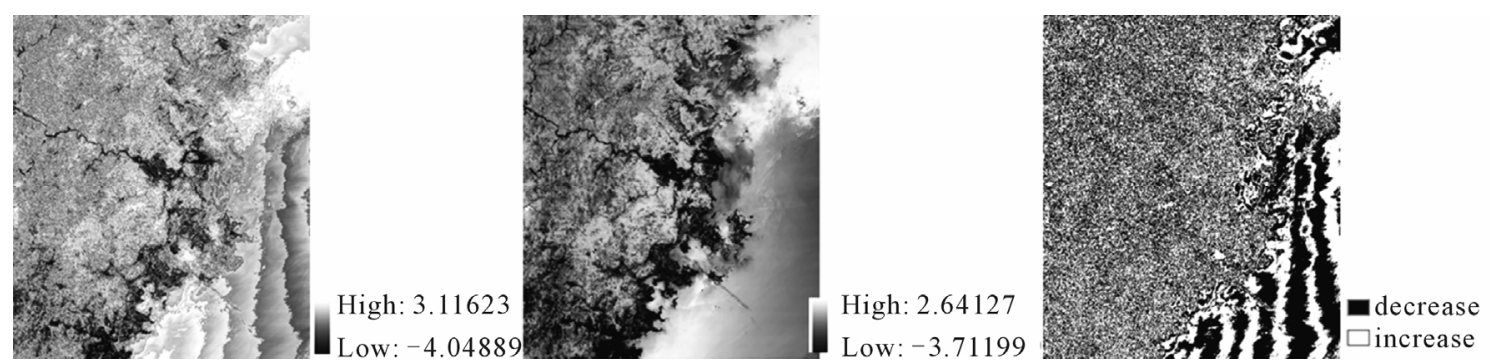

Figure 5. Difference image between the original and Antunes's 6S corrected image (left), the difference image between the original and improved $6 \mathrm{~S}$ corrected image (middle) and the difference image between the two 6S corrected images (right).

Table 1. Statistics of the images.

\begin{tabular}{lcccc}
\hline \multirow{2}{*}{ Images } & \multicolumn{3}{c}{ Statistics } \\
\cline { 2 - 5 } & Min (\%) & Max (\%) & Mean (\%) & Std (\%) \\
\hline original image & 3.605567 & 48.95111 & 7.313808 & 2.27023 \\
Antunes's 6S corrected image & 1 & 53 & 5.413397 & 2.68078 \\
improved 6S corrected image & 0.964300 & 52.66310 & 5.384489 & 2.69217 \\
difference image between the original and the Antunes's 6S & -4.048885 & 3.116226 & 1.900412 & 0.50168 \\
difference image between the original and the improved 6S & -3.711987 & 2.641267 & 1.929320 & 0.42220 \\
difference image between the improved 6S and Antunes's 6S & -0.696700 & 0.775700 & -0.028908 & 0.31402 \\
\hline
\end{tabular}

were used to compare the images before and after atmospheric correction. In addition, the statistics was computed and shown in Table 1.

From Figure 4, it is clear that the pixel value distribution of the image corrected by Antunes's $6 \mathrm{~S}$ model is concentrated, which means that there are a large number of pixels have the some reflectance range, while the other intervals have few pixels. The histogram of the image corrected by the improved $6 \mathrm{~S}$ model is closer to the histogram of the original image. So the distribution of pixel values is more uniform than that corrected by Antunes's $6 \mathrm{~S}$ model. It also can be seen that the range of pixel values becomes wide after atmospheric correction.

From the left and the middle difference images in Figure 5, it can be seenthat the changes between the improved $6 \mathrm{~S}$ corrected image and the original image were homogeneous, while the changes were sharp between Antunes's 6S corrected image and the original image. What's more, the two corrected images are different due to the different water vapor content. The influences made by the water vapor content can obviously see from the right image in Figure 5.

From Table 1, we can summarize that 1) in comparison with the original image, the pixel value range of the corrected images (both the two 6S codes) extends and the mean value decreases sharply while the standard deviation grows slightly; 2) after atmospheric correction, the mean value of the two corrected images decreases by
1.900412 and 1.929320 , respectively; 3 ) there are a lot of changes of the statistics between the two corrected images, but the mean value decrease by 0.028909 after atmospheric correction using the improved $6 \mathrm{~S}$ code.

\section{Conclusions}

The atmospheric correction $6 \mathrm{~S}$ code was improved by reading the water vapor content retrieved from MODIS data with $1 \mathrm{~km}$ resolution. After atmospheric correction using the improved $6 \mathrm{~S}$ model, the pixel value range of the image extended. Compared with the image corrected by Antunes's $6 \mathrm{~S}$ model, the pixel value is more successive and the histogram was closer to the histogram of the original image. Therefore, the improved $6 \mathrm{~S}$ model is more reasonable than the Antunes's 6S model in atmospheric correction.

Though this improved $6 \mathrm{~S}$ code was only applied to MODIS, it can be applied to other satellite data. Validation need to be done and the aerosol optical thickness need to be considered in this code. Further work is ongoing.

\section{Acknowledgements}

This work was supported by the Natural Science Foundation for Distinguished Young Scholars of Fujian Province (Grant No. 2009J06024). We also thank Mauro A. Homem Antunes for his extended 6S code. 


\section{REFERENCES}

[1] B. Cairns and B. E. Carlson, "Atmospheric Correction and Its Application to an Analysis of Hyperion Data," IEEE Transaction on Geoscience and Remote Sensing, Vol. 41, No. 6, 2003, pp. 1232-1245.

doi:10.1109/TGRS.2003.813134

[2] E. F. Vermote, D. Tanre, J. L. Deuze, M. Herman, and J.-J. Morcrette, "Second Simulation of the Satellite Signal in the Solar Spectrum, 6S: An Overview," IEEE Transactions on Geoscience and Remote Sensing, Vol. 35, No. 3, 1997, pp. 675-686. doi:10.1109/36.581987

[3] B. Y. Wu, W. Li, H. B. Chen, F. Li, W. X. Zhang and D. Lu, "Practical Algorithms of Atmospheric Radiative Transfer," Meteorological Press, Beijing, 1998, pp. 21-40 (in Chinese).

[4] S. L. Liang, H. L. Fang and M. Z. Chen, "Atmospheric Correction of Landsat ETM+ Land Surface Imagery-Part I: Method," IEEE Transactions on Geoscience and Remote Sensing, Vol. 39, No. 11, 2001, pp. 2490-2498. doi: $10.1109 / 36.964986$
[5] Y. J. Kaufman and B. C. Gao, "Remote Sensing of Water Vapor in the Near IR from EOS/MODIS," IEEE Transactions on Geosciences and Remote Sensing, Vol. 30, No. 5, 1992, pp. 871-884. doi:10.1109/36.175321

[6] J. A. Sobrino and J. E. Kharraz, "Surface Temperature and Water Vapor Retrieval from MODIS Data," International Journal of Remote Sensing, Vol. 24, No. 24, 2003, pp. 5161-5182. doi: 10.1080/0143116031000102502

[7] K. B. Mao, H. D. Li, D. Y. Hu, J. Wang, J. X. Huang, et al., "Estimation of Water Vapor Content in Near-Infrared Bands around $1 \mu \mathrm{m}$ from MODIS Data by Using RMMN," Optics Express, Vol. 18, No. 9, 2010, pp. 95429554. doi:10.1364/OE.18.009542

[8] W. J. Zhao, M. Tamura and H. Takahashi, "Atmospheric and Spectral Corrections for Estimating Surface Albedo from Satellite Data Using 6S Code," Remote Sensing of environment, Vol. 76, No. 2, 2000, pp. 202-212. doi:10.1016/S0034-4257(00)00204-2

[9] 6S User Guide Version 2, 1997. 